\title{
Appropriateness and cost-effectiveness in the treatment of invasive candidiasis in Internal Medicine Wards
}

\author{
Enrico Tagliaferri, Francesco Menichetti \\ UOC Malattie Infettive, AOU Pisana, Pisa, Italy
}

\begin{abstract}
Invasive candidiasis (IC), including candidemia, is a major cause of morbidity and mortality among patients and the majority of cases of candidemia are documented in Medical Wards. Early identification of patients at risk, knowledge of local epidemiology and prompt efforts to define etiologic diagnosis are pivotal to ensure appropriateness. Start with an echinocandin and switch to fluconazole when possible, seems to represent a useful strategy for the management of IC. The choice between the three echinocandins should be based on the specific indications, pharmacokinetic/pharmacodynamic profile, clinical experience and cost.
\end{abstract}

\section{Invasive candidiasis}

Invasive candidiasis (IC), including candidemia, is a major cause of morbidity and mortality among patients undergoing to major surgery, receiving broad spectrum antibiotic therapy, having severe clinical conditions, multiple co-morbidities and indwelling devices. ${ }^{1,2}$

Today the majority of cases of candidemia are documented in Medical Wards. ${ }^{3}$ In our tertiary-care, 800 beds, University Hospital, $50 \%$ of the total 222 episodes of candidemia documented in the period 2012-2013, were observed in Medical Wards. The distribution of the remaining episodes was $27 \%$ (60 episodes) in surgical wards, $18 \%$ (41) in Intensive Care Unit (ICU), 5\% (11) in other wards.

Candida albicans was responsible for 50\% (122 episodes) of total cases of candidemia while the nonalbicans species were represented by Candida parapsilosis 27\% (65), Candida glabrata 12\% (30), Candida tropicalis 5\% (13) and Candida krusei 2.5\% (6).

C. glabrata has a dose-dependent susceptibility to

Correspondence: Francesco Menichetti, UOC Malattie Infettive, AOU Pisana, Pisa, Italy.

Tel.: +39.050995431 - Fax: +39.050995471.

E-mail: menichettifrancesco@gmail.com

Key words: antifungals, invasive candidiasis, Internal Medicine Wards.

Received for publication: 29 July 2014

Accepted for publication: 30 July 2014.

This work is licensed under a Creative Commons Attribution NonCommercial 3.0 License (CC BY-NC 3.0).

CCopyright E. Tagliaferri and F. Menichetti, 2014

Licensee PAGEPress, Italy

Italian Journal of Medicine 2014; 8:221-224

doi:10.4081/itjm.2014.536 fluconazole while C. krusei has an innate resistance to the azoles. ${ }^{4} C$. parapsilosis, often associated with central venous catheter infections, is less susceptible in vitro to the echinocandins. Amphotericin B has a good antifungal activity against all isolates of $C$. albicans and virtually all the non-albicans species.

The outcome of candidemia seems to be closely related to Candida species: fungemia with a more favorable outcome are those caused by C. parapsilosis, followed by those due to C. albicans, C. glabrata, $C$. tropicalis and C. krusei. ${ }^{5}$

Therefore the knowledge of local epidemiology plays a pivotal role in planning an appropriate empirical treatment for Candida infections.

Timely and appropriate antifungal therapy is crucial for patient outcome: any delay is associated with an increase of mortality. ${ }^{6}$

Another independent variable associated with mortality is represented by the production of biofilm, the extracellular matrix produced by microorganisms in particular conditions, typically in the presence of foreign bodies such as indwelling devices and prosthesis. ${ }^{7}$ Azoles lack any significant activity against biofilm while echinocandins show variable efficacy; amphotericin B shows in vitro a consistent activity. ${ }^{8}$

Treating infections due to biofilm-forming Candida with highly active anti-biofilm antifungal agents like caspofungin seems to favorably influence patient survival with respect to fluconazole therapy. ${ }^{9}$

To ensure a timely antifungal treatment, an empirical, fever-driven approach is often used..$^{10}$ This strategy, although largely adopted in the setting of patients with hematologic malignancies, did not show consistent efficacy in ICU patients. Schuster et al. ${ }^{11}$ compared high-dose intravenously fluconazole $(800 \mathrm{mg})$ to placebo in persistently febrile ICU patients not responding to antibacterial therapy. Quite unexpectedly, no difference was documented between the two treat- 
ment groups. Two possible explanations for this result were represented by the intrinsic limit of fluconazole antifungal activity and by the patient population unselected for high risk of IC.

Several predictive algorithms and score systems have been proposed to identify patients at higher risk of candidemia and invasive candidiasis. The Candida score by León et al. ${ }^{12}$ is based on total parenteral nutrition (one point), recent surgery (one point), multifocal Candida colonization (one point), and severe sepsis (two points). With a score of 3 or greater, the relative risk of developing fungemia and invasive candidiasis is increasing and the start of empirical antifungal therapy is justified. However, this score has been validated in the Surgery and ICU setting and may be less useful for patients cared for in Medical Wards.

Systematic efforts to obtain an etiologic diagnosis have to be made to ensure an appropriate treatment. Culture methods still play a key role in the diagnosis of IC. However, sensitivity of blood culture in case of candidemia is around 50\%. Recently Tascini et al. showed that cultures taken from arterial blood presented a shorter time to positivity (TTP) with respect to blood cultures drawn from peripheral vein; it is noteworthy that the time sparing was around $12 \mathrm{~h}$. However, no significant difference was documented in TTP for arterial blood and blood drawn from central venous catheter. ${ }^{13}$

Serological methods for the diagnosis of invasive candidiasis include the combined detection of mannan antigen and anti-mannan antibodies and the $\beta$-glucan antigen. Mannan is a genus specific antigen produced by Candida in the early stages of the infection, but a lythic enzyme clears it rapidly from the serum. Sensitivity may be increased by the concomitant search of the anti-mannan antibodies that, becoming positive at a later stage, are more longer detectable. ${ }^{14}$

The $\beta$-glucan is a panfungal test useful for the detection of Candida, Aspergillus and Pneumocystis jiroveci. The test has a low specificity due to several causes of false positive results. Trend in the betaglucan levels seems to be useful in predicting the outcome of invasive candidiasis and the response to antifungal therapy. ${ }^{15}$

Significant advances have also been made in polymerase chain reaction methods for rapid detection of Candida in blood specimens but further evaluation of this approach in different clinical settings is needed. ${ }^{16}$

Guidelines for the treatment of IC has been proposed by the Infectious Diseases Society of America (IDSA) in $2009^{4}$ and by the European Society of Clinical Microbiology and Infectious Diseases (ESCMID $)^{10}$ in 2012. Both guidelines emphasize the role of echinocandins; the ESCMID guidelines also suggest a severe downgrading for fluconazole and conventional amphotericin $B$.
Recently, an Italian consensus for IC management has been issued (ITALIC). ${ }^{17}$ The authors focused on patient stratification in terms of risk factors for IC and clinical severity. According to the ITALIC, echinocandins are indicated as first-line treatment of IC because of fungicidal activity, activity against strains producing biofilms, activity against fluconazole-resistant strains, favorable safety profile and low propensity for drug-drug interactions.

The choice between the three echinocandins should be based on the specific indications, pharmacokinetic/pharmacodynamic profile, clinical experience and cost.

Caspofungin, the first echinocandin introduced in the Italian market, is the most widely used echinocandin with a large amount of data related to the efficacy and safety profile; anidulafungin lacks any metabolism (bio-degradation) and may be useful in patients with severe liver disease; micafungin, although limited by the European Medicines Agency warning, is indicated for neonates.

As an alternative, for critically ill patients, the use of liposomal amphotericin B may be considered; voriconazole should be reserved for selected cases.

In stable patients, fluconazole may represent a possible choice.

In case of Candida endophtalmitis, pyelonephritis and meningitis, voriconazole is the drug of choice.

Patients should be treated for at least 14 days after the last positive blood culture and even more in deepseated infections.

De-escalation from an echinocandin to intravenous or oral fluconazole should be encouraged when the patient is clinically stable and the isolated strain is susceptible.

Intravascular non-surgical catheters should be removed in all patients with documented catheter-related fungemia.

Main characteristics of antifungals are summarized in Table 1.

The choice of an antifungal therapeutic strategy has a deep impact on the hospital epidemiology: Lortholary et al. reported that the extensive use of caspofungin decrease the isolation of $C$. albicans from $56 \%$ to $21 \%$ and increase C. glabrata from $18 \%$ to $35 \%$ and $C$. parapsilosis from $13 \%$ to $31 \% .{ }^{18}$ Similar epidemiological modifications were observed using fluconazole. The risk of infection with an isolate with decreased susceptibility to fluconazole or caspofungin is associated with the recent exposure to these drugs. ${ }^{19}$

A major concern for the extensive use of echinocandins is represented by cost.

Cost is definitely higher with respect to fluconazole but similar to the cost of true competitors. In particular echinocandins cost more than voriconazole but less than liposomal amphotericin B. 
Table 1. Summary of the main characteristics of antifungal agents for treating invasive candidiasis/candidemia.

\begin{tabular}{lccc}
\hline & Fluconazole & Echinocandins & Amphotericin B \\
\hline Spectrum of activity & Limited & Wide & Wide \\
\hline Antifungal activity & Fungistatic & Usually fungicidal & Fungicidal \\
\hline Anti-biofilm activity & Low & Variable & Consistent \\
\hline Safety & Good & Very good & Nephrotoxicity \\
\hline Evidence from RCTs & Inferior to echinocandins & Good & Adequate \\
\hline Guidelines ECCMID & CI & AI & BI (liposomal) \\
\hline Cost & Very low & Medium & Very high (liposomal)
\end{tabular}

RCTs, randomized controlled trials; ECCMID, European Congress of Clinical Microbiology and Infectious Diseases.

Despite a higher cost, echinocandins (aniludafungin, micafungin) have demonstrated to reduce mortality and overall in-hospital costs compared to fluconazole, both in the setting of empirical and definite treatment of IC. ${ }^{20-22}$ Notably, some authors suggest that caspofungin is more cost-effective than fluconazole in the empiric treatment of IC when fluconazole resistance is higher than $25 \%$ in hospital, reinforcing the importance of knowing the local epidemiology. ${ }^{23}$

No difference between micafungin and caspofungin has been demonstrated in terms of cost-effectiveness in the treatment of candidemia and IC. ${ }^{24}$

Caspofungin has been proved to be more cost effective than liposomal amphotericin B in the empirical treatment of invasive fungal infections and the treatment of candidemia, not only for a lower cost of drug, but also for a lower incidence of renal failure, ${ }^{25,26}$ similar data have been reported for micafungin. ${ }^{27}$

De-escalation strategy (initial treatment with echinocandins, followed by fluconazole when possible) has been proved to reduce mortality and improve the outcome of IC with a significant cost saving, compared to escalation strategy (initial treatment with fluconazole).$^{28}$

However, a timely start of a broad-spectrum antifungal with fungicidal and anti-biofilm activity, switching to a cheaper alternatives according to microbiology results and clinical status is considered to be the main determinant of cost-effectiveness, regardless the antifungal agent. ${ }^{29}$

In conclusion, IC is a relevant problem also in Medical Wards.

Early identification of patients at risk, knowledge of local epidemiology and prompt efforts to define etiologic diagnosis are pivotal to ensure appropriateness. Start with an echinocandin and switch to fluconazole when possible, seems to represent a useful strategy for the management of IC.

\section{References}

1. Tortorano AM, Peman J, Bernhardt H, et al. Epidemiology of candidaemia in Europe: results of 28-month European Confederation of Medical Mycology (ECMM) hospital-based surveillance study. Eur J Clin Microbiol Infect Dis 2004;23:317-22.

2. Eggimann P, Bille J, Marchetti O. Diagnosis of invasive candidiasis in the ICU. Ann Intens Care 2011;1:37.

3. Bassetti M, Merelli M, Righi E, et al. Epidemiology, species distribution, antifungal susceptibility, and outcome of candidemia across five sites in Italy and Spain. J Clin Microbiol 2013;51:4167-72.

4. Pappas PG, Kauffman CA, Andes D, et al. Clinical practice guidelines for the management of candidiasis: 2009 update by the Infectious Diseases Society of America. Clin Infect Dis 2009;48:503-35.

5. Horn DL, Neofytos D, Anaissie EJ, et al. Epidemiology and outcomes of candidemia in 2019 patients: data from the prospective antifungal therapy alliance registry. Clin Infect Dis 2009;48:1695-703.

6. Morrell M, Fraser VJ, Kollef MH. Delaying the empiric treatment of candida bloodstream infection until positive blood culture results are obtained: a potential risk factor for hospital mortality. Antimicrob Agents Chemother 2005;49:3640-5.

7. Tumbarello M, Posteraro B, Trecarichi EM, et al. Biofilm production by Candida species and inadequate antifungal therapy as predictors of mortality for patients with candidemia. J Clin Microbiol 2007;45:1843-50.

8. Choi HW, Shin JH, Jung SI, et al. Species-specific differences in the susceptibilities of biofilms formed by Candida bloodstream isolates to echinocandin antifungals. Antimicrob Agents Chemother 2007;51:1520-3.

9. Tumbarello M, Fiori B, Trecarichi EM, et al. Risk factors and outcomes of candidemia caused by biofilm-forming isolates in a tertiary care hospital. PLoS One 2012; 7:e33705.

10. Cornely OA, Bassetti M, Calandra T, et al. ESCMID* guideline for the diagnosis and management of Candida diseases 2012: non-neutropenic adult patients. Clin Microbiol Infect 2012;18:19-37. 
11. Schuster MG, Edwards JE Jr, Sobel JD, et al. Empirical fluconazole versus placebo for intensive care unit patients: a randomized trial. Ann Intern Med 2008;149: 83-90.

12. León C, Ruiz-Santana S, Saavedra P, et al. Usefulness of the 'Candida score' for discriminating between Candida colonization and invasive candidiasis in non-neutropenic critically ill patients: a prospective multicenter study. Crit Care Med 2009;37:1624-33.

13. Tascini C, Sbrana F, Cardinali G, et al. Arterial blood culture to hasten the diagnosis of candidemia in critically ill patients. Intensive Care Med 2014;40:1059-60.

14. Mikulska M, Calandra T, Sanguinetti M, et al. The use of mannan antigen and anti-mannan antibodies in the diagnosis of invasive candidiasis: recommendations from the Third European Conference on Infections in Leukemia. Crit Care 2010;14:R222.

15. Jaijakul S, Vazquez JA, Swanson RN, Ostrosky-Zeichner L. (1,3)- $\beta$-D-glucan as a prognostic marker of treatment response in invasive candidiasis. Clin Infect Dis 2012; 55:521-6.

16. Ahmad S, Khan Z. Invasive candidiasis: a review of nonculture-based laboratory diagnostic methods. Indian J Med Microbiol 2012;30:264-9.

17. Scudeller L, Viscoli C, Menichetti F, et al. An Italian consensus for invasive candidiasis management (ITALIC). Infection 2014;42:263-79.

18. Lortholary O, Desnos-Ollivier M, Sitbon K, et al. Recent exposure to caspofungin or fluconazole influences the epidemiology of candidemia: a prospective multicenter study involving 2,441 patients. Antimicrob Agents Chemother 2011;55:532-8.

19. Blanchard E, Lortholary O, Boukris-Sitbon K, et al. Prior caspofungin exposure in patients with hematological malignancies is a risk factor for subsequent fungemia due to decreased susceptibility in Candida spp.: a case-control study in Paris, France. Antimicrob Agents Chemother 2011;55:5358-61.

20. Neoh CF, Liew D, Slavin M, et al. Cost-effectiveness analysis of anidulafungin versus fluconazole for the treatment of invasive candidiasis. J Antimicrob Chemother 2011; 66:1906-15.

21. Grau S, Salavert M, Carlos Pozo Laderas J, et al. Costeffectiveness of anidulafungin in confirmed candidaemia and other invasive Candida infections in Spain. J Mycol Med 2013;23:155-63.

22. Zilberberg MD, Kothari S, Shorr AF. Cost-effectiveness of micafungin as an alternative to fluconazole empiric treatment of suspected ICU-acquired candidemia among patients with sepsis: a model simulation. Crit Care 2009;13:R94.

23. Golan Y, Wolf MP, Pauker SG, et al. Empirical anti-Candida therapy among selected patients in the intensive care unit: a cost-effectiveness analysis. Ann Intern Med 2005;143:857-69.

24. Neoh CF, Liew D, Slavin MA, et al. Economic evaluation of micafungin versus caspofungin for the treatment of candidaemia and invasive candidiasis. Intern Med J 2013;43:668-77.

25. Turner SJ, Senol E, Kara A, et al. Pharmacoeconomic evaluation of caspofungin versus liposomal amphotericin $B$ in empirical treatment of invasive fungal infections in Turkey. Int J Antimicrob Agents 2013;42:276-80.

26. Wingard JR, Wood CA, Sullivan E, et al. Caspofungin versus amphotericin B for candidemia: a pharmacoeconomic analysis. Clin Ther 2005;27:960-9.

27. Cornely OA, Sidhu M, Odeyemi I, et al. Economic analysis of micafungin versus liposomal amphotericin $\mathrm{B}$ for treatment of candidaemia and invasive candidiasis in Germany. Curr Med Res Opin 2008;24:1743-53.

28. Masterton RG, Casamayor M, Musingarimi P, et al. Deescalation from micafungin is a cost-effective alternative to traditional escalation from fluconazole in the treatment of patients with systemic Candida infections. J Med Econ 2013;16:1344-56.

29. Wilke M. Treatment and prophylaxis of invasive candidiasis with anidulafungin, caspofungin and micafungin and its impact on use and costs: review of the literature. Eur J Med Res 2011;16:180-6. 